\title{
Análise comparativa entre métodos alternativo e convencional para amostras de mosquitos obtidos a partir de habitats fitotélmicos (Bromeliaceae) na Floresta Atlântica, Serra do Mar, Paraná, Brasil ${ }^{1}$
}

\author{
Ana Leuch Lozovei ${ }^{2}$ \\ Mário Antônio Navarro da Silva ${ }^{3}$
}

\begin{abstract}
Comparison between alternative and usual methods to study mosquitoes samples from bromeliads habitat (Bromeliaceae), Atlantic Forest, Sea Mountain ridge, Paraná, Brazil. The collects from mosquitoes' sample that reproduce in leaf imbricate of bromeliads were done by two differents methods: a conventional method where the plants were felled and the water inside were dropped in a recipient and in the second method the samples were collected by sucking the water, so the plant wasn't felled. The number of bromeliads studied in each method was 120 plants from zero to $15 \mathrm{~m}$ height. The period of study lasted from january till december 1989. The bromeliads were collected monthly by each method. The score of immature was done at the laboratory. The variables studied were the number of leaf axils with water $\left(\mathrm{X}_{1}, \mathrm{Y}_{1}\right)$, water volume $\left(\mathrm{cm}^{3}\right)$ inside each bromeliad $\left(\mathrm{X}_{2}, \mathrm{Y}_{2}\right)$, the number of immature mosquitoes (larvae and pupae) inside each water volume from each bromeliad $\left(\mathrm{X}_{3}, \mathrm{Y}_{3}\right)$, respectivelly, for the methods that the samples were obtained: by sucking or by felling the plant and the time (month) variable. By this study, we concluded that there is no significative difference between the methods done for $\alpha=0,05$. There is also no significative difference between both methods in each month. However, there was significative difference between the months for $\alpha=0.05$.

KEY WORDS. Diptera, Culicidae, bromeliads, sampling methods
\end{abstract}

Bromélias, plantas monocotiledôneas da família Bromeliaceae, usualmente crescem dispondo-se em forma de roseta formada pelas imbricações de suas folhas próximo à raiz. Entre as imbricações acumula-se água de chuva, formando, assim, um reservatório em que proliferam diversas espécies de mosquitos, além de outra fauna. PADILla (1973), estudioso deste grupo de plantas, relatou que as bromélias desenvolvem-se no solo em terrenos pedregosos e principalmente em troncos de árvores. Tanto as terrestres como as epífitas, são muito numerosas em florestas abertas e preservadas de climas tropicais e subtropicais úmidos das Américas, estendendo-se desde o nível do mar até as altitudes acima de $1.200 \mathrm{~m}$. Sua distribuição geográfica estende-se desde a região Sul dos Estados Unidos até aproximadamente $800 \mathrm{~km}$ perto do extremo sul da Argentina.

1) Contribuição número 1148 do Departamento de Zoologia, Universidade Federal do Paraná.

2) Universidade Federal do Paraná, Departamento de Patologia Básica, Centro Politécnico. Caixa Postal 19031, 81531-990 Curitiba, Paraná, Brasil.

3) Departamento de Zoologia, Universidade Federal do Paraná. Caixa Postal 19020, 81531-990 Curitiba, Paraná, Brasil. 
A capacidade das bromélias em reter volume de água por longo tempo é um recurso natural para a sua própria sobrevivência. Esta água acumulada contém substâncias minerais que são absorvidas pelas folhas da planta. Os mosquitos com seus recursos próprios localizam-nas e detectam que isto lhes favorece as condições para proliferação.

Até o momento, todos os estudos para a obtenção de amostras de mosquitos imaturos em bromélias utilizaram-se do método convencional - destruindo as plantas, isto é, arrancando-as pela raiz. Como este método provoca a erradicação de bromélias, danificando a flora, surgiu a idéia de testar uma nova modalidade (método) de coleta mais fácil e menos drástica. $\mathrm{O}$ método em teste, no presente trabalho, consiste na preservação das plantas nas amostragens de mosquitos imaturos por sucção. Foi testado, analisado e está sendo divulgado pela primeira vez.

A literatura existente apresenta as coletas de amostras de mosquitos bromelícolas somente pelo método convencional, conhecido desde o século passado e que foi publicado por LUTZ (1950). Entre outros pesquisadores, podem ser citados DowNS \& PITTENDRIGH (1946) que se utilizaram desta metodologia para coletar imaturos no estudo da relação bromélias-malária em Trinidad; PITTENDRIGH (1950), no estudo de Anopheles (Kerteszia) bellator Dyar \& Knab, 1906 associado à malária endêmica em plantações de cacau em Trinidad; RACHOU \& RicciarDI (1951) e RACHOU et al. (1954a,b) nos estudos, respectivamente, da distribuição geográfica de anofelineos no Brasil, da dispersão de Anopheles (Nyssorhynchus) darlingi Root, 1926, do recrudescimento epidêmico da malária e da distribuição dos anofelíneos do Paraná; Veloso \& CALÁBria (1953) e Veloso et al. (1956a,b) no estudo de mosquitos bromelícolas do subgênero Kerteszia Theobald, 1905 no sul do Brasil, Santa Catarina; ANDRADE \& BRANDÃo (1957) e ANDRADE \& VERANO (1957) em investigação relacionada com a distribuição geográfica e incidência da fauna de anofelíneos no Espírito Santo e em Goiás; ARAGÃo (1968a,b,c) nas pesquisas relacionadas com o ciclo anual de várias espécies de Anopheles (Kerteszia) em bromeliáceas; TORALES et al. (1972) utilizaram-se desta técnica convencional, para obter amostras de mosquitos bromelícolas em Corrientes na Argentina. Na Califórnia, Estados Unidos, FrANK et al. $(1976,1977)$ empregaram o método para estudar, respectivamente, os imaturos de Wyeomyia (Wyeomyia) vanduzeei Dyar \& Knab, 1906 em imbricações de bromélias e os fatores de controle do tamanho das populações de Wyeomyia (Wyeomyia) medioalbipes Lutz, 1904 e Wyeomyia vanduzeei relacionando-as com o tamanho das bromélias e o número de estádios imaturos. FRANK \& CURTIS (1977a,b) serviram-se da metodologia no estudo de Wyeomyia vanduzeei e Wyeomyia medioalbipes, bromelícolas, para verificar em ambas as espécies a capacidade de evolução em condições limitadas e intermitentes de suplemento alimentar e para estimar a correlação do número de larvas e pupas que proliferam em bromélias versus precipitações pluviométricas.

No Brasil, recentemente, pesquisadores também utilizaram-se da mesma metodologia para investigar mosquitos bromelícolas, como DORVILLÉ (1995) no estudo da composição e aspectos da biologia e da fauna de mosquitos na restinga do Rio de Janeiro, inclusive nas formas bromelícolas. MurILlo et al. (1988), na costa do Pacífico na Colômbia, também serviram-se do método convencional para coletar formas imaturas de Anopheles (Kerteszia) neivai Dyar \& Knab, 1913 em 
bromélias com o objetivo de estudar a flutuação da população larval e as características dos criadouros bromelícolas.

Para obter dados e análises confiáveis na avaliação das amostragens efetuadas pelos dois métodos distintos, no presente trabalho, procurou-se obter determinado número de amostras pareadas de mosquitos bromelícolas, observando os critérios de equivalência em tamanhos e alturas de fixação vertical das bromélias, assim como o número de plantas amostradas e também, a obtenção de dados das variáveis pré-estabelecidas - número de imbricações com água, volume de água e o número de imaturos (larvas e pupas) por planta, além da variável tempo (meses do ano).

\section{MATERIAL E MÉTODOS}

O presente trabalho foi desenvolvido na Floresta Atlântica, Serra do Mar, no Município de Quatro Barras no Estado do Paraná, Brasil, área compreendida entre $25^{\circ} 26^{\prime} 49^{\prime \prime} \mathrm{S}, 49^{\circ} 13^{\prime} 54^{\prime \prime} \mathrm{W}$ e $910 \mathrm{~m}$ de altitude.

O período de investigação foi estendido entre janeiro e dezembro de 1989. As amostras de mosquitos imaturos foram obtidas de zero a $15 \mathrm{~m}$ de altura em bromélias (Bromeliaceae), plantas que acumulam água das chuvas formando assim os criadouros para diversas espécies de mosquitos. As amostras pareadas, mensais, de mosquitos imaturos foram coletadas concomitantemente pelos dois métodos distintos, nas mesmas condições, datas, número igual de plantas amostradas, de tamanho o mais próximo possível e nas mesmas alturas. Os procedimentos para obter estas amostras de mosquitos imaturos em bromélias seguiram dois métodos distintos. O primeiro, o de obter as amostras por sucção mecânica da água de entre as axilas foliares para dentro de um frasco de vidro hermeticamente fechado, dotado de dois furos na rolha de borracha onde se inseriram dois tubos de polietileno, bem ajustados para formar um vácuo no interior do frasco da água, preservando as plantas intactas que continham os mosquitos; o segundo, o método convencional (LUTZ 1950) em que apararam-se as folhas das bromélias próximo às suas imbricações foliares logo acima do acúmulo da água, em seguida, destacaram-se as plantas pela raiz para invertê-las dentro de um recipiente a fim de receber a água com mosquitos. Antes, porém, para ambos os métodos, contava-se em cada bromélia amostrada o número de axilas foliares com água, dado este que constituía uma das variáveis a serem analisadas. O recipiente usado para recolher as amostras de ambas as técnicas foi uma bandeja esmaltada branca com dimensões de $45 \times 35 \times 5,5 \mathrm{~cm}$. Para garantir o aproveitamento total do material biológico disponível, as imbricações foliares eram lavadas com a própria água recolhida da planta.

Ao recolher as amostras da bandeja para os recipientes de plástico de $500 \mathrm{ml}$ com tampa, procedia-se a mensuração do volume de água com uma proveta de 500 $\mathrm{ml}$ constituindo, assim, outra variável a ser considerada. Todos estes dados foram anotados em uma planilha apropriada, assim como o número de cada bromélia e a data das coletas. As amostras de mosquitos bromelícolas foram recolhidas em espécies de bromélias mais abundantes na área de estudo, predominantemente em Aechmea (Pothuava) cylindrata Lindm., 1891 e Vriesea (Vriesea) platynema Gaud, 1843. 
No laboratório, procedia-se a contagem do número de mosquitos imaturos (larvas e pupas) por bromélia, variável que é também mensurada no presente trabalho. Portanto, as variáveis analisadas comparativamente para ambos os métodos foram: o número de axilas foliares com água por planta $\left(\mathrm{X}_{1}, \mathrm{Y}_{1}\right)$; o volume de água $\left(\mathrm{cm}^{3}\right)$ por planta $\left(\mathrm{X}_{2}, \mathrm{Y}_{2}\right)$ o número de mosquitos por planta $\left(\mathrm{X}_{3}, \mathrm{Y}_{3}\right)$, onde $\mathrm{X}_{1}, \mathrm{X}_{2}$ e $\mathrm{X}_{3}$ correspondem às variáveis para o método das amostras obtidas por sucção (teste), enquanto $\mathrm{Y}_{1}, \mathrm{Y}_{2} \mathrm{e} \mathrm{Y}_{3}$, para o método convencional. E ainda a variável tempo em período-meses das coletas de amostras.

Para cada um dos métodos foram amostradas 120 bromélias, tendo 1635 imbricações com água e imaturos para o método em teste e 1614 imbricações, para o método tradicional. No total foram 240 plantas de alturas variáveis, porém, pareadas em cada amostra. A variável "altura" foi observada para pareamento das amostras, mas não foi avaliada.

Variáveis mensuradas. A fim de poder comparar os métodos em teste e o convencional de uma maneira padronizada que levasse em conta o número de axilas foliares, o número de mosquitos imaturos e o volume de água, calcularam-se as variáveis $\mathbf{Z}_{\mathbf{A}}=\mathbf{X}_{\mathbf{3}} / \mathbf{X}_{\mathbf{1}} \mathrm{e} \mathbf{Z}_{\mathbf{B}}=\mathbf{Y}_{\mathbf{3}} / \mathbf{Y}_{\mathbf{1}}$, que medem o número de mosquitos imaturos por imbricação foliar com água e também as variáveis $\mathbf{W}_{\mathbf{A}}=\mathbf{X}_{\mathbf{3}} / \mathbf{X}_{\mathbf{2}} \mathrm{e} \mathbf{W}_{\mathbf{B}}=\mathbf{Y}_{3} / \mathbf{Y}_{2}$, que medem o número de mosquitos imaturos por centímetro cúbico de água.

\section{Tipo de Modelo para a análise de variância}

Como é desejado comparar os métodos dentro de cada mês (evitando eventuais variações sazonais na diferenciação das técnicas), trata-se de um modelo hierárquico. Ainda, como os resultados dizem respeito aos dois métodos especificados (e não às várias técnicas que eventualmente existam ou venham a existir), o modelo hierárquico é do tipo fixo.

\section{Especificação do Modelo de análise}

Seguindo o acima considerado, o modelo pode ser especificado (tanto para as variáveis " $Z$ " como para as "W", segundo JOHNSON \& LEONE (1964):

$\mathbf{X}_{t, i, j}=\mathbf{A}+\mathbf{M}_{\mathbf{t}}+\mathbf{S}_{t}, \mathbf{i}+\mathbf{Z}_{t}, \mathbf{i}, \mathbf{j}$, onde: $\left(\mathbf{X}_{t}, \mathbf{i}, \mathbf{j}\right)$ valor observado (número de mosquitos imaturos por imbricação foliar com água ou por centímetro cúbico de água) na j-ézima observação, do i-ézimo método, t-ézimo mês; $(\mathbf{A})$ média geral; $\left(\mathbf{M}_{\mathbf{t}}\right)$ desvio médio com relação à A no $\mathrm{t}$-ézimo mês $(\mathrm{t}=1,2 \ldots \ldots \ldots 12) ;\left(\mathbf{S}_{\mathbf{t}}, \mathrm{i}\right)$ desvio médio com relação a A + Mt para i-ézimo método, no t-ézimo mês $(i=1,2$ e t $=1,2, \ldots \ldots \ldots \ldots . . .12)$; $\left(\mathbf{Z}_{t}, \mathrm{i}, \mathrm{j}\right)$ resíduo para a j-ézima observação, no i-ézimo método, t-ézimo mês $(\mathrm{t}=$ $1,2, \ldots \ldots \ldots . . .12 ; i=1,2 ; j=1,2, \ldots \ldots \ldots . .10)$.

\section{Pressupostos para a análise de variância}

Será pressuposto como usual em análises de variâncias: a) esperança matemática de $Z_{t}, i=0$ para todo t e todo $i$; b) $Z_{t}$, i são variáveis aleatórias mutuamente independentes, normalmente distribuídas.

\section{Teste preliminar}

O pré-requisito para a análise - homogeneidade das variâncias - foi verificado através do teste de Cochran ao nível de 5\% (DIXON \& MASSEY 1957). 


\section{Testes realizados pela análise de variâncias}

$\mathrm{O}$ teste de que $\mathrm{M}_{1}=\mathrm{M}_{2}+\mathrm{M}_{3}=\ldots \ldots . . \mathrm{M}_{12}=0$ (igualdade das médias entre os meses) foi realizado pelo teste $\mathrm{F}$ através da comparação do quadrado médio "entre meses" com o quadrado médio residual.

$\mathrm{O}$ teste de que $\mathrm{S}_{\mathrm{t}, \mathrm{i}}=0$ para todo t e todo i (diferença entre os métodos, em cada mês) foi realizado pelo teste $\mathrm{F}$ através da comparação do quadrado médio "entre métodos dentro de meses" com o quadrado médio dos resíduos.

Os testes mencionados foram realizados ao nível de significância de 5\%.

\section{Contrastes}

Para as variáveis cujos testes realizados através de análise de variância mostraram diferenças significativas ao nível de $5 \%$, procedeu-se a realização dos contrastes de SCHEFFÉ (1953) ao mesmo nível.

O procedimento consiste em selecionar médias dessas variáveis para as quais podem ser relevantes comparações individuais ou do tipo média de médias.

Um contraste entre médias $\mathrm{M}_{1}, \mathrm{M}_{2}, \ldots \ldots . . \mathrm{M}_{12}$ é definido como: $\theta_{\mathbf{o b}}=\Sigma \lambda_{\mathbf{i}} \cdot \mathbf{m}_{\mathbf{i}}$, $\operatorname{com} \Sigma \lambda_{\mathbf{i}}=\mathbf{0}$.

A título de ilustração, por exemplo, se é desejado comparar a média do terceiro mês ( $\left.\mathrm{m}_{3}\right)$ com a do quinto mês ( $\left.\mathrm{m}_{5}\right)$, os $\lambda_{\mathrm{i}}$ serão $\lambda_{3}=1, \lambda_{5}=-1, \lambda_{\mathrm{i}}=0$ para os demais meses.

Ainda, a título de exemplo hipotético, o contraste que compara a média das médias dos seis primeiros meses com a média das médias dos seis últimos será:

$\lambda_{\mathrm{i}}=1(\mathrm{i}=1,2, \ldots 6) ; \lambda_{\mathrm{i}}=-1(\mathrm{i}=7,8, \ldots 12)$.

Os valores dos contrastes observados $\theta_{\mathbf{o b}}$ são comparados com o valor crítico de $\theta_{\mathbf{c}}$, dado por:

$$
\Theta_{c}=\left\{(k-1) F_{k, v, \alpha} \cdot S^{2} \cdot \sum_{i=1}^{k} \frac{\lambda_{i}^{2}}{n_{i}}\right\}^{1 / 2}
$$

onde: (k) número de médias que podem ser comparadas; $\left(\mathbf{F}_{\mathbf{k}, \mathbf{v}, \alpha)}\right.$ ) percentual $95 \mathrm{da}$ distribuição " $F$ " com " $k$ " graus de liberdade no numerador e " $v$ " graus de liberdade no denominador (no presente caso, $v=2 / 6$ - número graus de liberdade residual); $\left(\mathbf{S}^{2}\right)$ quadrado médio residual; $\left(\lambda_{\mathrm{i}}\right)$ número de observações da i-ézima média.

Para analisar os métodos empregados com recursos estatísticos, estabeleceram-se as seguintes hipóteses: $\mathrm{H} 0$ ) os dois métodos equivalem-se nas amostragens de mosquitos bromelícolas; H1: os dois métodos apresentam diferenças nas amostragens de mosquitos bromelícolas.

Para os dados combinados de ambos os métodos através de médias mensais únicas: H0) não existem diferenças sazonais nas amostras coligidas; H1) existem diferenças sazonais entre os meses trabalhados.

\section{RESULTADOS E DISCUSSÃO}

Os resultados para a análise dos dois métodos em questão constam em cinco tabelas. As tabelas I e II contêm as médias, as variâncias e os desvios padrões das variáveis z e w. Nas tabelas III e IV, estão estabelecidas as análises de variâncias 
para as variáveis z e w. Os contrastes para detectar quais os meses que provocaram as diferenças significativas a nível de $5 \%$, onde foram combinados os resultados de ambos os métodos através de médias mensais encontram-se na tabela $\mathrm{V}$.

Tabela I. Número médio de mosquitos imaturos, larvas e pupas, por imbricações foliares de bromélias, Floresta Atlântica, Serra do Mar, Quatro Barras, Paraná.

\begin{tabular}{|c|c|c|c|c|c|c|}
\hline \multirow{2}{*}{ Meses } & \multicolumn{2}{|c|}{ Médias } & \multicolumn{2}{|c|}{ Variâncias } & \multicolumn{2}{|c|}{ Desvios padrăo } \\
\hline & $\begin{array}{l}\text { Método em } \\
\text { teste }\end{array}$ & $\begin{array}{c}\text { Método } \\
\text { convencional }\end{array}$ & $\begin{array}{l}\text { Método em } \\
\text { teste }\end{array}$ & $\begin{array}{c}\text { Método } \\
\text { convencional }\end{array}$ & $\begin{array}{l}\text { Método em } \\
\text { teste }\end{array}$ & $\begin{array}{c}\text { Método } \\
\text { convencional }\end{array}$ \\
\hline Janeiro & 2,923 & 2,197 & 0,947 & 0,596 & 0,973 & 0,772 \\
\hline Fevereiro & 2,458 & 1,735 & 3,763 & 0,779 & 1,940 & 0,883 \\
\hline Março & 2,725 & 1,496 & 2,245 & 0,585 & 1,498 & 0,765 \\
\hline Abril & 1,871 & 2,058 & 0,633 & 0,326 & 0,796 & 0,571 \\
\hline Maio & 2,220 & 2,011 & 1,219 & 3,369 & 1,104 & 1,836 \\
\hline Junho & 2,478 & 2,467 & 1,800 & 3,384 & 1,342 & 1,840 \\
\hline Julho & 1,498 & 1,689 & 0,571 & 1,021 & 0,755 & 1,011 \\
\hline Agosto & 1,939 & 1,106 & 2,898 & 0,251 & 1,702 & 0,501 \\
\hline Setembro & 1,426 & 1,422 & 1,325 & 0,272 & 1,151 & 0,521 \\
\hline Outubro & 1,105 & 1,030 & 1,651 & 0,226 & 1,285 & 0,475 \\
\hline Novembro & 1,390 & 1,824 & 0,491 & 0,426 & 0,701 & 0,653 \\
\hline Dezembro & 2,374 & 3,065 & 0,915 & 3,358 & 0,957 & 1,833 \\
\hline Total & 2,034 & 1,842 & 1,712 & 1,408 & 1,309 & 1,187 \\
\hline
\end{tabular}

Tabela II. Número médio de mosquitos imaturos, larvas e pupas, por centímetro cúbico de água em bromélias, Floresta Atlântica, Serra do Mar, Quatro Barras, Paraná.

\begin{tabular}{|c|c|c|c|c|c|c|}
\hline \multirow{2}{*}{ Meses } & \multicolumn{2}{|c|}{ Médias } & \multicolumn{2}{|c|}{ Variâncias } & \multicolumn{2}{|c|}{ Desvios padrăo } \\
\hline & $\begin{array}{l}\text { Método em } \\
\text { teste }\end{array}$ & $\begin{array}{c}\text { Método } \\
\text { convencional }\end{array}$ & $\begin{array}{l}\text { Método em } \\
\text { teste }\end{array}$ & $\begin{array}{c}\text { Método } \\
\text { convencional }\end{array}$ & $\begin{array}{l}\text { Método em } \\
\text { teste }\end{array}$ & $\begin{array}{l}\text { Método } \\
\text { convencional }\end{array}$ \\
\hline Janeiro & 0,117 & 0,086 & 0,002 & 0,001 & 0,049 & 0,033 \\
\hline Fevereiro & 0,126 & 0,081 & 0,020 & 0,002 & 0,141 & 0,049 \\
\hline Março & 0,144 & 0,078 & 0,008 & 0,001 & 0,088 & 0,025 \\
\hline Abril & 0,163 & 0,238 & 0,016 & 0,021 & 0,126 & 0,146 \\
\hline Maio & 0,148 & 0,178 & 0,010 & 0,023 & 0,100 & 0,153 \\
\hline Junho & 0,155 & 0,136 & 0,009 & 0,007 & 0,097 & 0,086 \\
\hline Julho & 0,107 & 0,065 & 0,003 & 0,002 & 0,054 & 0,039 \\
\hline Agosto & 0,096 & 0,062 & 0,006 & 0,003 & 0,077 & 0,050 \\
\hline Setembro & 0,095 & 0,054 & 0,015 & 0,001 & 0,123 & 0,036 \\
\hline Outubro & 0,035 & 0,027 & 0,001 & 0,000 & 0,035 & 0,013 \\
\hline Novembro & 0,094 & 0,114 & 0,006 & 0,021 & 0,080 & 0,146 \\
\hline Dezembro & 0,099 & 0,066 & 0,001 & 0,001 & 0,030 & 0,033 \\
\hline Total & 0,115 & 0,099 & 0,009 & 0,010 & 0,092 & 0,098 \\
\hline
\end{tabular}

No teste de homogeneidade de variância com os valores das tabelas acima referidas, calculou-se o valor de:

$$
C=\frac{S_{i, j_{\text {maximu }}}^{2}}{\sum_{i} \sum_{j} S_{i}^{2}}
$$

onde: $(\mathbf{C})$ homogeneidade; $\left(\mathrm{S}_{\mathrm{i}}{ }^{2}\right)$ é a variância observada no mês “i”, método “j”, resultando em: $\mathrm{C}=0,114$ para a variável "z" (número de imaturos/imbricação); $\mathrm{C}=0,128$ para a variável $\mathrm{w}$ (número de imaturos/centímetro cúbico de água). O valor 
crítico de C é 0,136 (DiXON \& MASSEY 1957). Portanto, conclui-se não haver diferença significativa entre as variâncias.

Tabela III. Número de mosquitos imaturos, larvas e pupas, por imbricação foliar com água em bromélias, Floresta Atlântica, Serra do Mar, Quatro Barras, Paraná.

\begin{tabular}{|c|c|c|c|c|c|}
\hline Fonte de variaçăo & Graus de liberdade & Soma dos quadrados & Quadrado médio & "F" observado & "F" crítico (5\%) \\
\hline Entre meses & 11 & 55,467 & 5,042 & $3,656^{\star}$ & 1,79 \\
\hline Entre métodos & 12 & 20,193 & 1,683 & 1,220 & 1,75 \\
\hline Resíduo & 216 & 297,861 & 1,379 & & \\
\hline Total & 239 & 373,521 & & & \\
\hline
\end{tabular}

$\left(^{\star}\right)$ Valor significante a $5 \%$.

Nas análises de variâncias para as variáveis z e w que constam nas tabelas III e IV, observou-se em ambos os casos que: a) não haver diferenças significantes entre os métodos em cada um dos meses e ainda; b) haver diferenças significantes entre os meses.

Tabela IV. Número de mosquitos imaturos, larvas e pupas, por centímetro cúbico de água em imbricações foliares de bromélias, Floresta Atlântica, Serra do Mar, Quatro Barras, Paraná.

\begin{tabular}{|c|c|c|c|c|c|}
\hline Fonte de variaçăo & Graus de liberdade & Soma dos quadrados & Quadrado médio & "F" observado & "F" crítico (5\%) \\
\hline Entre meses & 11 & 0,443 & 0,040 & $5,00^{*}$ & 1,79 \\
\hline Entre métodos & 12 & 0,101 & 0,008 & 1,00 & 1,75 \\
\hline Residuo & 216 & 1,633 & 0,008 & & \\
\hline Total & 239 & 2,177 & & & \\
\hline
\end{tabular}

$\left({ }^{\star}\right)$ Valor significante a $5 \%$.

Tabela V. Médias mensais de mosquitos imaturos, larvas e pupas, por imbricação foliar com água e número de imaturos por centímetro cúbico de água em bromélias, de ambos os métodos, Floresta Atlântica, Serra do Mar, Quatro Barras, Paraná.

\begin{tabular}{lcc}
\hline \multicolumn{1}{c}{ Meses } & Imaturos/folhas com água & Imaturos $/ \mathrm{cm}^{3}$ de água \\
\hline Janeiro & 2,560 & 0,102 \\
Fevereiro & 2,096 & 0,103 \\
Março & 2,111 & 0,111 \\
Abril & 1,965 & 0,201 \\
Maio & 2,120 & 0,163 \\
Junho & 2,472 & 0,145 \\
Julho & 1,593 & 0,086 \\
Agosto & 1,514 & 0,079 \\
Setembro & 1,424 & 0,074 \\
Outubro & 1,067 & 0,031 \\
Novembro & 1,607 & 0,104 \\
Dezembro & 2,720 & 0,082 \\
\hline Total & 1,938 & 0,107 \\
\hline
\end{tabular}

De posse dos resultados das análises de variância pode-se aplicar contrastes de SCHEFFÉ (1953) a fim de detectar quais os meses que provocaram as diferenças significativas. Já que não houve diferenças entre os métodos em questão, pode-se 
reunir os resultados de ambos os métodos combinando-os através de médias mensais únicas cujos valores constam na tabela $\mathrm{V}$. Na comparação entre cada mês, os valores críticos dos contrastes resultaram em: $\theta_{\mathrm{c}}=1,629$ para variável "número de mosquitos imaturos por imbricação foliar" $\mathrm{e} \theta_{\mathrm{c}}=0,124$ para a variável "número de imaturos por centímetro cúbico de água". Disto depreende-se que os únicos meses cujas médias diferem significativamente são dezembro e outubro para a variável "número de imaturos por imbricação foliar", sendo a diferença de média igual a 1,653; abril e outubro com diferença média de 0,170 e ainda, abril e setembro sendo de 0,127 a diferença de médias para a variável "número de imaturos por centímetro cúbico de água".

Também foram verificados os meses cujas médias estavam acima da média geral para observar se diferem significativamente daqueles meses com médias abaixo da média geral em conjunto. Para a variável "número de imaturos por imbricação foliar" com o que os meses são: de janeiro a junho e dezembro com médias acima da média geral $(1,938)$, enquanto de julho a novembro, com médias abaixo da média geral.

Para a variável "número de imaturos por centímetro cúbico de água", os meses com médias maiores da geral são os de março a junho, enquanto todos os demais, com médias menores da média geral.

No primeiro caso, os contrastes são do tipo: $\{1 / 7,1 / 7,1 / 7,1 / 7,1 / 7,1 / 7,1 / 7$, $-1 / 5,-1 / 5,-1 / 5,-1 / 5,-1 / 5\}$ e no segundo caso, apresentam-se assim: $\{1 / 4,1 / 4,1 / 4$, $1 / 4,-1 / 8,-1 / 8,-1 / 8,-1 / 8,-1 / 8,-1 / 8,-1 / 8,-1 / 8\}$, resultando nos valores observados dos contrastes $2,292-1,441=0,851$ e $0,155-0,083=0,072$, respectivamente. Os valores críticos dos contrastes são: $\theta_{\mathrm{c}}=0,675$ para a variável "número de imaturos/imbricação foliar"; e $\theta_{c}=0,137$ para a variável "número de imaturos/centímetro cúbico de água".

Concluindo, portanto, para a variável "número de imaturos por imbricação foliar com água", os meses cujas médias são maiores que a média geral têm média comum significativamente maior que a média comum dos demais meses. E para a variável "número de imaturos por centímetro cúbico de água", a média comum dos meses cujas médias individuais são superiores à média geral do ano não difere significativamente da média comum dos demais meses.

Percebe-se da análise efetuada que os dois métodos empregados para amostrar mosquitos bromelícolas equivalem-se na eficiência de obter amostras de imaturos. O método em teste neste trabalho, o de obtenção de amostras por sucção, é de mais fácil execução no campo, exige menos equipamentos e, ainda, as amostras são obtidas rapidamente em alguns minutos, além de preservar as plantas intactas respeitando, assim, o equilíbrio da Natureza. Este mesmo processo de sucção com grandes vantagens pode ser utilizado para controlar a proliferação de insetos indesejáveis em bromeliáceas nos jardins e ambientes domiciliares.

AGRADECIMENTOS. Ao Dr. Gert Guenther Hatschbach, chefe do Museu Botânico Municipal de Curitiba, pela identificação das bromélias. Ao Dr. Jair Lício Ferreira Santos, Professor de Estatística, Faculdade de Saúde Pública, USP, São Paulo pela execução da análise estatística. 


\section{REFERÊNCIAS BIBLIOGRÁFICAS}

ANDRADE, R.M. \& H. BRANDÃo. 1957. Contribuição para o Conhecimento da Fauna de Anofelinos do Estado do Espírito Santo. Área de Distribuição e Incidência das Espécies por Cidades, Vilas e Povoados (Diptera, Culicidae). Rev. Bras. Malar. Doenças Trop. 9 (3): 391-399.

ANDRADE, R.M. \& O.T. VERANO. 1957. Contribuição para o Conhecimento da Fauna de Anofelinos do Estado de Goiás. Área de Distribuição e Incidência das Espécies por Sedes Municipais e Distritais, Povoados e Fazendas (Diptera, Culicidae). Rev. Bras. Malar. Doenças Trop. 9 (3): 366-390.

ARAGÃo, M.B. 1968a. O Ciclo Anual dos Anopheles do Subgênero Kerteszia no Sul do Brasil. Mem. Inst. Oswaldo Cruz 66 (1): 85-106.

1968b. Sobre a Distribuição Vertical dos Criadouros de Anopheles do Subgênero Kerteszia no Sul do Brasil. Mem. Inst. Oswaldo Cruz 66 (2): 132-144.

- $1968 \mathrm{c}$. Sobre a Biomassa dos Anopheles do Subgênero Kerteszia em Seus Criadouros. Mem. Inst. Oswaldo Cruz 66 (2): 227-237.

DiXON, W.J. \& F.J. MASSEY. 1957. Introduction to Statistical Analysis. New York, McGrow Hill, 488p.

DorVILLÉ, L.F.M. 1995. Composição e Aspectos da Biologia da Fauna de Mosquitos (Diptera, Culicidae) da Restinga de Barra de Maricá, (R.J.). Revta bras. Ent. 39 (1): 203-219.

DownS, W.G. \& C.S. PitTENDRIGH. 1946. Bromeliad Malaria in Trinidad, British West Indies. Amer. Jour. Trop. Med. 26: 47-66.

FranK, J.H. \& G.A. CURTIS. 1977a. On the Bionomics of Bromeliad-Inhabiting Mosquitoes. III. The Probable Stratezy of Larval Feeding in Wyeomyia vanduzeei and Wy. medioalbipes. Mosq. News 37 (2): 200-206.

1977b. On the Bionomics of Bromeliad-Inhabiting Mosquitoes. IV. Egg Mortality of Wyeomyia vanduzeei caused by Rainfall. Mosq. News 37 (2): 239-245.

Frank, J.H.; G.A. CURTIS \& H.T. EVANS. 1976. On the Bionomics of BromeliadInhabiting Mosquitoes. I. Some Factors Influencing Oviposition by Wyeomyia vanduzeei. Mosq. News 36 (1): 25-30.

1977. On the Bionomics of Bromeliad-Inhabiting Mosquitoes. II. The relationship of Bromeliad Size to the Number of Immature Wyeomyia vanduzeei and Wy. medioalbipes. Mosq. News 37 (2): 180-192.

Johnson, N.L. \& F. LeONE. 1964. Statistical and Experimental Design. New York, Johan Wiley, Vol. 2, $2^{\text {nd }}$ ed., 399 p.

LuTz, A. 1950. Mosquitos da Floresta e Malária Silvestre. Rev. Bras. Malar. Doenças Trop. 2 (2): 91-100.

Murillo, B.C.; V.R. Astaiza \& O.P. Fajardo. 1988. Biologia de Anopheles (Kerteszia) neivai H., D \& K. 1913 (Diptera: Culicidae) en la Costa Pacífica de Colombia. I. Fluctuación de la Población Larval y Caracteristicas de sus criaderos. Rev. Saúde Públ., São Paulo, 22 (2): 94-100.

PADIlla, V. 1973. Bromeliads. New York, Crown Publishers Inc., 143p.

PitTEndRIGH, C.S. 1950. The Quantitative Evaluation of Kerteszia Breeding Grounds. Amer. Jour. Trop. Med. 30: 457-468. 
RACHOU, R.G. \& I. RicCiARDI. 1951. Contribuição ao Conhecimento da Distribuição Geográfica dos Anofelinos no Brasil: Estado do Paraná (Distribuição por Municípios e Localidades). Rev. Bras. Malar. Doenças Trop. 3: 423-447.

RACHOU, R. G.; A.G.S. LôBo \& E. LuZ. 1954a. Dispersão do Anopheles (N.) darlingi no Recrudescimento Epidêmico de Malária em 1950 no Norte do Paraná. Rev. Bras. Malar. Doenças Trop. 6 (3): 411-414.

—.1954b. Atualização da Distribuição Geográfica dos Anofelinos do Estado do Paraná. Rev. Bras. Malar. Doenças Trop. 6 (4): 525-532.

SCHEFFÉ, H.A. 1953. Method for Judging all Contrast in Analysis of Variance. Biometrika 40: 87-104.

ToRales, G.J.; W.H. HACK \& B. TURN. 1972. Criaderos de Culicidos en Bromeliaceas del NW de Corrientes. Act. Zool. Lilloana 29: 293-308.

Veloso, H.P. \& P.V. CALÁBRIA. 1953. O problema Ecológico Vegetação Bromeliáceas e Anofelinos. II. Avaliação quantitativa dos criadouros e das formas aquáticas dos Anofelinos do subgênero Kerteszia nos principais tipos de vegetação do Município de Brusque, Estado de Santa Catarina. Sollowia Na. Bot. Herbário Barbosa Rodrigues 5: 7-36.

Veloso, H.P.; P. FonTANA JR. \& R.M. KLEIN. 1956a. Os anofelinos do subgênero Kerteszia em relação à distribuição das bromeliáceas em comunidades florestais do município de Brusque, Estado de Santa Catarina. Mem. Inst. Oswaldo Cruz 54 (1): 1-86.

Veloso, H.P.; J.V. Moura \& R.M. KLeIN. 1956b. Delimitação ecológica dos anofelineos do subgênero Kerteszia na região costeira do sul do Brasil. Mem. Inst. Oswaldo Cruz 54 (1): 517-548.

Recebido em 04.VI.1998; aceito em 26.V.1999. 